Hegemonia - Revista Eletrônica de Relações Internacionais do Centro Universitário Unieuro ISSN: 1809-1261

UNIEURO, Brasília, número 20, Janeiro-Junho de 2017, pp. 50-64.

Recebido em: 12/9/2016

Avaliado em: 28/9/2016

Aprovado em: 21/10/2016

\title{
Ecofeminismo: Un Pensamiento Ambientalista de Corte Feminista
}

\author{
María Alejandra Montilla Muñoz e Gerson Galo Ledezma Meneses ${ }^{2}$
}

Resumen: Este texto busco comprender el punto de encuentro entre el feminismo y la ecología, entendiendo lo que el ecofeminismo pretende al unificar las demandas del movimiento de las mujeres con el del movimiento ecológico. Teniendo en cuenta las similares formas de opresión, abordando la mirada desde América Latina, la cosmovisión indígena y la búsqueda por el empoderamiento.

Palabras - clave: Feminismo, ecología, ecofeminismo, mujeres.

Resumo: No texto procurou compreender o ponto de encontro entre o feminismo e ecologia, entendendo o que o ecofeminismo pretende ao unificar as demandas do movimento das mulheres com o do movimento ecológico. Tendo em conta as semelhantes formas de opressão, abordando o olhar desde a América Latina, a cosmovisão indígena, e a busca pelo empoderamento.

Palavras-chave: feminismo, ecologia, ecofeminismo, mulheres.

Abstract: This paper has tried to understand the point of encounter between feminism and ecology, understanding what ecofeminism aims to unify the demands of the women's movement with the ecological movement. Taking into account the similar forms of oppression, approaching the look from Latin America, the indigenous worldview, and the quest for empowerment.

Keywords: feminism, ecology, eco-feminism, women

\footnotetext{
${ }^{1}$ Estudiante de Maestría en Integración Contemporánea en América Latina, ICAL, Línea de Investigación: Integración, Cultura y Sociedad. Universidad Federal da Integración Latino-americana, UNILA. Dirección: Calle 26an 6b-26 Teléfono (57) 3003374652 correo electrónico: maria.munoz@unila.edu.co

2 Profesor de la Universidad Federal de la Integración Latinoamericana, Unila. gersonledezma@yahoo.com
} 
Hegemonia - Revista Eletrônica de Relações Internacionais do Centro Universitário Unieuro ISSN: $1809-1261$

UNIEURO, Brasília, número 20, Janeiro-Junho de 2017, pp. 50-64.

Introducción

A las reclamaciones de los derechos de las mujeres también se han sumado las luchas ambientales, el rol de las mujeres se ha concentrado además en la emergencia de un nuevo paradigma alternativo de relación con la naturaleza.

Esa naturaleza que a lo largo de nuestra historia como especie, el ser humano ha usado y dominado en beneficio propio, y la ha visto como un objeto para su uso y provecho, llegando a los límites de la sobreexplotación de los recursos; es así como en el tipo de relación asumida entre humano y naturaleza en la modernidad, el humano no se ve a sí mismo como parte de esta, sino superior a ella, esto conlleva a una visión de la naturaleza como una $\operatorname{cosa}^{3}$ y, por lo tanto, una destrucción de las condiciones que permiten la vida en la tierra. ${ }^{4}$

En ese sentido en épocas de cambio global, de crisis ambiental, de la invisibilidad de una sociedad patriarcal y del sistema económico capitalista, se ha generado que las visiones de feminismo y de los movimientos ecologistas, encuentren puntos en común, creando un nuevo movimiento social, el "ecofeminismo", definido como :

Un intento de abordar la cuestión medioambiental desde las categorías de mujeres, género, androcentrismo ${ }^{5}$ y sexismo, proponiendo opciones para lograr la sostenibilidad y, desde la mirada feminista, corregir los sesgos. (Puleo, 2008, p 42)

\footnotetext{
3 Cosificación: Reducir a la condición de cosa aquello que no lo es.

4 Merchant, C. The death of nature. En M. Zimmerman, J. Baird y G. Sessions. Environmental philosophy, Prentice Hall, Nueva Jersey, 1993 pp. 268-283.

5 Práctica de otorgar al punto de vista masculino una posición central en la propia visión del mundo, de la cultura y de la historia
} 
Hegemonia - Revista Eletrônica de Relações Internacionais do Centro Universitário Unieuro ISSN: 1809-1261

UNIEURO, Brasília, número 20, Janeiro-Junho de 2017, pp. 50-64.

Es relevante estudiar este tema pues el rol que juegan el ecofeminismo es significativo, ya que enriquece y concientiza en torno a una visión y acciones que están afectando tanto a la naturaleza como a los mismos humanos, pues dentro del ecofeminismo se rescata una corriente de pensamiento, con elementos fundamentales como la ecología y el feminismo como posición político-social ${ }^{6}$, fundamental para comprender el origen de la ideología androcéntrica predominante.

El objetivo de este escrito es comprender lo que el ecofeminismo pretende al unificar las demandas del movimiento de las mujeres con el del movimiento ecológico, analizando los puntos de encuentro de estos dos movimientos, para entender como las formas de interacción y apropiación de las mujeres se utilizan respecto al medio ambiente.

Así entonces, se dice que el ecofeminismo como consecuencia de la conexión entre el feminismo y la ecología se encuentra en las utopías literarias de las feministas de la década de 1970, se señala que la socióloga François D’eabuonne ${ }^{7}$ utilizó este término por primera vez para "definir las acciones desarrolladas por feministas francesas que protestaban la ocurrencia de un desastre ecológico”, y así criticar la modernidad desde dos disciplinas, el feminismo y el ecologismo, analizando la conexión ideológica existente entre la explotación de la naturaleza y la explotación de las mujeres.

Sin embargo, para algunos el ecofeminismo surgió al mismo tiempo que los movimientos ecologistas y ambientalistas de la década de 1960. Sin tenerle relevancia la fecha de su surgimiento exacto, el término incluye una diversidad de perspectivas sobre la naturaleza de las relaciones que se dan dentro de ella.

\footnotetext{
6 BEDREGAL, Teresa, Ecofeminismo, Biología y Teoría Feminista, Agenda de las mujeres, Argentina, 2005 pág 1 en 1978 el movimiento Ecología-Feminismo, que tuvo poco eco en París, pero tuvo más éxito en Australia y Estados Unidos.
} 
Hegemonia - Revista Eletrônica de Relações Internacionais do Centro Universitário Unieuro ISSN: $1809-1261$

UNIEURO, Brasília, número 20, Janeiro-Junho de 2017, pp. 50-64.

Se puede explicar que tradicionalmente al hablar de naturaleza, se hace referencia al soporte material de la vida, la vida no exclusivamente humana, sino también animal, vegetal; y hablar de cultura es hablar de sociedad humana.

Esto se ha controvertido de múltiples formas en las últimas décadas, primero que todo sobre esa visión antropocéntrica en que el hombre está frente a la naturaleza (de la cual harían parte las mujeres) y por encima de ella. El ser humano habría dominado a la naturaleza gracias a la cultura, por lo tanto cultura y naturaleza son los dos extremos de esta controversial relación de dominación/explotación que ha atravesado la visión patriarcal de Occidente. Pero ya los estudios feministas han dejado al descubierto la trama cultural que ha permitido siglos (tal vez milenios) de dominación masculina. ${ }^{8}$

Puesto que es notorio que dentro del ecofeminismo, la existencia de vínculos importantes entre la explotación, opresión y violencia contra las mujeres y contra la naturaleza, existan conexiones que se dan porque en los dos casos las explotaciones proceden del sistema patriarcal, y de una feminización de la naturaleza que trae como correlato una naturalización de las mujeres.

Ve en las jerarquías sociales del patriarcado la causa principal de la destrucción ecológica y del dominio de la mujer. El patriarcado aparece como la forma social que organiza el pensamiento, la cultura y las relaciones de género. Las cosmogonías y formas de uso de la naturaleza son más "ecológicas" en sociedades tradicionales. Mas no por ello las relaciones sociales son menos patriarcales e impera menos la gerontocracia y el dominio sobre la mujer. (Leff, 2004, p283)

Así también el ecofeminismo y la economía feminista destacan la semejanza entre la explotación de las mujeres y la de la naturaleza, a través del trabajo reproductivo

8 ULLOA, Astrid, Concepciones de la naturaleza en la antropología actual IN Cultura y Naturaleza: Aproximaciones a propósito del bicentenario de la independencia de Colombia, Leonardo Montenegro Martínez (Editor), Bogotá: Jardín Botánico de Bogotá, José Celestino Mutis, 2011. pág 325 
Hegemonia - Revista Eletrônica de Relações Internacionais do Centro Universitário Unieuro ISSN: 1809-1261

UNIEURO, Brasília, número 20, Janeiro-Junho de 2017, pp. 50-64.

invisibilizado, no reconocido, ni agradecido. Con esto se hace referencia a aquellas labores relacionadas a la reproducción humana, la crianza, la resolución de las necesidades básicas, la promoción de la salud, el apoyo emocional, la facilitación de la participación social, etc. ${ }^{9}$

Algunas autoras se asientan en las desigualdades internacionales para manifestar que existen diferentes ecofeminismos. Pues manifiestan que existe el ecofeminismo del Primer Mundo el cual se desarrollaría en un terreno más bien ideológico, y por otro lado, también el ecofeminismo del Tercer Mundo ${ }^{10}$, crecidamente pragmático, que parte de la dependencia del medio natural para la subsistencia de las mujeres vulnerables rurales pobres, campesinas, indígenas, y sus familias.

Dentro de este último ecofeminismo es de resaltar que en la India han surgido dos grandes corrientes, una de ellas por Bina Agarwal, dirigente del feminismo ecológico, con estudios teóricos y empíricos, con un enfoque particular en los más desfavorecidos y que propugna una forma mucho más "materna" de cuidar la vida, independientemente del género; y la otra por Vandana Shiva, defensora del ecofeminismo radical, quien ha sido una de las voces más influyentes del ecofeminismo en todo el mundo, su pensamiento se basa en la religión y la filosofía hindúes que describen el "principio femenino" como la fuente de vida y la base de un desarrollo sustentable.

Asimismo, critica el modelo económico dominante, ya que propaga las técnicas de plantación de monocultivos tanto en los bosques como en la agricultura; y reflexiona que el

9 LEÒN, Magdalena: Cambiar la economía para cambiar la vida. Desafíos de una economía para la vida, Alberto Acosta y Esperanza Martínez (comp.): El buen vivir, Una vía para el desarrollo, Abya Yala, Quito, 2009 pág 184

10 FERNANDEZ, Alex, Feminismo ecológico, Eroski Consumer, 2012 
Hegemonia - Revista Eletrônica de Relações Internacionais do Centro Universitário Unieuro ISSN: 1809-1261

UNIEURO, Brasília, número 20, Janeiro-Junho de 2017, pp. 50-64.

sistema económico indio tradicional preserva la relación mutua con la naturaleza a través del policultivo, cuyo objetivo es la producción de subsistencia local con insumos propios ${ }^{11}$.

Bina Agarwal sugiere un marco alternativo el cual llama ambientalismo feminista, reconoce que la relación entre el medio ambiente y las mujeres, está determinado por una estructura que entiende diversos aspectos, dentro de los cuales se incluye género, clase (casta/raza), distribución del ingreso, organización de la producción y reproducción ${ }^{12}$, ignoradas en algunas corrientes ecofeministas.

Al mismo tiempo, resalta que los procesos de degradación ambiental y de apropiación de los recursos naturales por unos cuantos tienen implicaciones específicas de clase, género y ubicación geográfica; esta autora afirma que "son las mujeres provenientes de áreas rurales y familias pobres las que resultan afectadas de manera más negativa y las que han participado más activamente en los movimientos ecológicos. Por lo tanto, explica también que no se puede considerar a las 'mujeres' como una categoría unitaria". ${ }^{13}$

Por el mismo camino, se encuentra la Australiana ecofeminista Ariel Salleh quien manifiesta que la existencia de las mujeres está ampliamente relacionada con los vínculos sociales que son parte de un contexto material; Salled plantea que un materialismo histórico ecofeminista investigue la relación que se encuentra entre las desigualdades biológicas de tanto hombres como mujeres y la construcción social que gira en torno de ellas.

\section{América Latina}

Ahora bien, pasemos al ecofeminismo en América Latina el cual constituye un movimiento relativamente reciente y que aún no se encuentra definido, pero que a pesar de eso ha

\footnotetext{
11 BRAIDOTTI, Rosi, "Mujeres, medio ambiente y desarrollo sustentable, Surgimiento del tema y diversas aproximaciones", en Verónica Vázquez García y Margarita Velázquez Gutiérrez (coords.), Miradas al futuro, PUEG/CRIM/CP, México, 1998. pp 24-30

12 AGARWAL, Bina, "El debate sobre género y medio ambiente: lecciones de la India", en Vázquez

García y Velázquez Gutiérrez, 1998. pág 239

13 Ibid
} 
Hegemonia - Revista Eletrônica de Relações Internacionais do Centro Universitário Unieuro ISSN: 1809-1261

UNIEURO, Brasília, número 20, Janeiro-Junho de 2017, pp. 50-64.

comenzado a conformarse en el transcurso de sus prácticas concretas para enfrentar las amenazas que sobre la vida de las mujeres y la de sus hijos/as ha significado el avance del capitalismo neoliberal, traducido éste en la implantación de modelos de producción y consumo lesivos para la naturaleza y para el ser humano, por ser altamente contaminantes y generadores de pobreza, lo que agrede directamente a las mujeres. ${ }^{14}$

Para la religiosa Brasileña, Ivone Gebara, la modernidad empieza con la redefinición del papel de las mujeres como amas de casa, subordinadas a las relaciones matrimoniales y a la familia. Al mismo tiempo la naturaleza, "liberada de la fuerza de los espíritus, despojada gradualmente de sus secretos, pasa a ser dominada por el espíritu científico masculino"15.

Manifiesta que el ecofeminismo como pensamiento y movimiento social se refiere básicamente a:

La conexión ideológica entre la explotación de las mujeres y la explotación de la naturaleza dentro del sistema jerárquicopatriarcal y, desde el punto de vista de la filosofía y la teología, lo considera como una sabiduría que pretende rescatar el ecosistema y las mujeres. (Gebara, 2000, p18)

En ese sentido, la filósofa feminista porteña, Alicia Puleo ${ }^{16}$ lo define como:

La corriente que asume la problemática ecológica como algo que puede ser abordado de manera pertinente en clave de género, aportando ciertas claves de comprensión de relación humana con la naturaleza. (Duque, 2009)

Muestra que uno de los puntos de contacto que hacen que la ecología sea un tema que importe más a las mujeres es la cuestión de la salud. Los trastornos debidos a la

14 COVA, Santana Nancy, El Ecofeminismo Latinoamericano, Las Mujeres y la Naturaleza como Simbolos, Cifra Nueva, Universidad de los Andes-Trujillo, 2005 pág 38

15 Gebara, Ivone, "Intuiciones ecofeministas, ensayo para repensar el conocimiento y la religión", Editorial Trotta, Madrid, 2000, pág 18

16 Filósofa feminista radicada en España. Ha destacado por el desarrollo del pensamiento ecofeminista. Entre sus principales publicaciones se encuentra Ecofeminismo para otro mundo posible 
Hegemonia - Revista Eletrônica de Relações Internacionais do Centro Universitário Unieuro ISSN: 1809-1261

UNIEURO, Brasília, número 20, Janeiro-Junho de 2017, pp. 50-64.

contaminación afectan particularmente a las mujeres porque su organismo tiene mayor proporción de tejido graso y algunos tóxicos se fijan en la grasa. Aunque el daño ambiental afecta a hombres y mujeres, son ellas las más afectadas ${ }^{17}$.

Esto nos hace entender que el pensamiento ecofeminista también en América Latina toma como referencia buena parte del pensamiento ecologista sobre el dualismo como causa de la objetivación de la naturaleza y dominación de las mujeres que conduce a la crisis ambiental, ${ }^{18}$ extendiendo la diferencia de género desde su origen biológico-simbólico hasta su construcción socio histórico.

\section{Cosmovisión Indígena}

Ahora bien, para hablar de las mujeres indígenas se asume que dentro de los pueblos indígenas en general la perspectiva respecto a la naturaleza es relativamente igual, no cambia, siendo hombres, mujeres o cualquiera de sus integrantes tienden a tener una visión holística de la naturaleza, así entonces se ven como seres humanos pertenecientes a la Tierra, y no como ajenos a ella.

Así entonces comprenden a la tierra como una entidad fértil y viva que tiene una cuantía exclusiva, y no utilitaria; no puede ser una materia pasiva e inanimada que deba ser explotada para la expansión comercial ni el supuesto avance económico.

17 ESPINOZA, Frida, "Mujer y Naturaleza: definiciones de Ecofeminismo, “ In, Planeta Capital, Revista Rufián, vol. No 10, Santiago de Chile, 2012 pág 1

18 LEFF, Enrique, El Retorno del Orden Simbólico, Racionalidad Ambiental, La reapropiación social de la naturaleza, Siglo XI Editores, 2004, pág 284 
Hegemonia - Revista Eletrônica de Relações Internacionais do Centro Universitário Unieuro ISSN: $1809-1261$

UNIEURO, Brasília, número 20, Janeiro-Junho de 2017, pp. 50-64.

La tierra para la mayoría de los indígenas tampoco es un paisaje bonito o un lugar para escaparse el fin de semana, por lo contrario para ellos su relación con la naturaleza es de respeto, puesto que es su guía, su fuente de vida.

Desde la década de 1970, la relación pueblos indígenas y naturaleza ha sido el centro del debate del ambientalismo en general, y el ecofemismo en particular, dentro del cual las mujeres indígenas ocupan un lugar relevante ${ }^{19}$. Los pueblos indígenas han ganado reconocimiento como actores ecológicos en las representaciones, discursos y políticas nacionales e internacionales, donde se asume que una espiritualidad y sensibilidad femenina puede ser encontrada en sus tradiciones.

Una de las representaciones que sustenta la relevancia de los pueblos indígenas en lo ambiental es su relación con la de la Madre Naturaleza o Madre Tierra (Pachamama). Esta relación se consolidó, en el contexto internacional, con el Convenio de Diversidad Biológica $(\mathrm{CDB})^{20}$, las discusiones de acceso a los recursos genéticos y conocimientos tradicionales, el capítulo 24 de la Agenda 21 y el Plan de acción mundial para la conservación y utilización sostenible de los recursos genéticos de las plantas para la alimentación y agricultura, en el marco de la FAO, entre otros, donde se reconoce el papel de las mujeres en la biodiversidad y la necesidad de la participación de las mismas.

Si bien estas asociaciones tienen muchas implicaciones para los indígenas ${ }^{21}$ han permitido a las mujeres indígenas consolidar redes como la Red Internacional de Mujeres Indígenas sobre Biodiversidad y acciones en torno a lo ambiental.

19 ULLOA, Astrid, Participación política y ambiental, Mujeres indígenas: dilemas de género y etnicidad en los escenarios latinoamericanos, Universidad Nacional de Colombia, Bogotá, 2007, pág 221

20 Los objetivos son "la conservación de la biodiversidad, el uso sostenible de sus componentes y la participación justa y equitativa de los beneficios resultantes de la utilización de los recursos genéticos.

$21 \quad$ ULLOA, Astrid, La construcción del nativo ecológico, Complejidades, paradojas y dilemas de la relación entre los movimientos indígenas y el ambientalismo en Colombia, Instituto Colombiano de Antropología e Historia, Colciencias, Bogotá, 2004, pág 320 
Hegemonia - Revista Eletrônica de Relações Internacionais do Centro Universitário Unieuro ISSN: 1809-1261

UNIEURO, Brasília, número 20, Janeiro-Junho de 2017, pp. 50-64.

Para finalizar quisiera agregar que en varios países, pero en este escrito traemos a colación Colombia $^{22}$ los conocimientos de la mayoría de las mujeres indígenas están basados en complejas y diversas nociones de naturaleza, articuladas con su tradición histórica, étnica, social y cultural. Dentro de estas concepciones se considera la relación ser humanonaturaleza como un proceso dinámico, en el que la naturaleza es un ente vivo y con ella se establecen relaciones de reciprocidad.

Es así como las definiciones y acciones asociadas a los conocimientos indígenas ${ }^{23}$ no se pueden desligar de la concepción de interdependencia entre la naturaleza y raza humana, ellas en su gran mayoría representan el deseo de retornar a un mundo ideal, a un estilo de vida preindustrial y a un mundo ecológicamente sostenible de autosubsistencia.

Búsqueda para el empoderamiento

Una de las formas en que las mujeres han ido ganando poder ha sido, primero, a través del asociacionismo o conformación de asociaciones de mujeres locales y, luego, de encuentros puntuales pero masivos de mujeres para discutir sus problemáticas. Un ejemplo de esto último fue el primer encuentro celebrado en mayo de 2009 en el propio cantón Muisne denominado "Primer Encuentro: mujeres del ecosistema manglar del Ecuador, nuestros sueños, nuestros derechos, nuestros retos".

22 Colombia reconoce numerosas poblaciones indígenas. Se estima un total de 701.860 habitantes (incluso 800.000 habitantes) que ocupan el 24\% del territorio nacional y que hablan más de 65 idiomas y 300 dialectos étnicos dispersos en los 32 departamentos. Colombia modificó su constitución política en 1991, en la que entraron en vigor una serie de leyes referidas a los pueblos indígenas pues, anteriormente, la población indígena se encontraba regulaba por la Ley 89 de 1890.

23 ESCOBAR, Elsa, ESCOBAR, Pía, PAZMIÑO, Aracely, ULLOA, Astrid (Editoras), Las mujeres indígenas en los escenarios de la biodiversidad, UICN Fundación Natura Colombia, ICANH, 2005, pág 21 
Hegemonia - Revista Eletrônica de Relações Internacionais do Centro Universitário Unieuro ISSN: 1809-1261

UNIEURO, Brasília, número 20, Janeiro-Junho de 2017, pp. 50-64.

Más de 80 mujeres compartieron este encuentro, en el que contaron sus historias como mujeres que enfrentan discriminación y violencia. En este encuentro se unieron mujeres de Colombia, Honduras, México y Brasil. "Concheras, cangrejeras, pescadoras, ostioneras, almejeras, mujeres compañeras del manglar en su trabajo, en su lucha por sobrevivir, reflexionaron de dónde vienen y hacia dónde van”.

Pintaron sus historias y la biodiversidad del manglar, pintaron sus familias trabajando y jugando en el manglar. También pintaron la destrucción y la reforestación. Pintaron cómo querían que fuera la vida en el futuro. Conversaron, se hicieron más amigas, empezaron a encontrarse con otras mujeres como ellas y a encontrarse con ellas mismas. (VEUTHEY, 2009)

Y reconstruyeron la memoria histórica de las mujeres que siempre han estado en el manglar.

Consideraciones finales

Como se vio, los conocimientos sobre los movimientos de las mujeres en relación con la naturaleza son prácticamente desconocidos pero son claves para que el movimiento ecofeminista forme un desarrollo sostenible, las mujeres, en medio de estas luchas, están obteniendo un protagonismo cada vez más notorio, sus roles de liderazgo cada vez son mayores.

En la actualidad la relación de los seres humanos con la naturaleza no es para nada ejemplar hemos hecho uso y abuso de los recursos naturales en pos de una idea de confort y egoísmo en total desmedida para beneficio propio, ya es hora de ejercer respeto sobre la naturaleza. 
Hegemonia - Revista Eletrônica de Relações Internacionais do Centro Universitário Unieuro ISSN: 1809-1261

UNIEURO, Brasília, número 20, Janeiro-Junho de 2017, pp. 50-64.

Definitivamente el ecologismo y el feminismo tienen muchos puntos en común, luchas metas que alcanzar, sus luchas por la visibilidad y reivindicación es algo que ha sido históricamente subordinado pero que poco a poco va dando fruto, por una mejor calidad de vida para provecho de toda la sociedad y de la madre naturaleza.

Para conseguir un cambio hacia una sociedad mejor se debe hacer una mudanza integral en los pensamientos y acciones de la gente en general; es decir, eliminar el machismo que el sistema patriarcal impuso, comprender que tanto las mujeres como los hombres gozamos exactamente de los mismos derechos, sabiendo que no hay razón alguna para considerar a un ser superior o inferior a otro y esto mismo sobre la naturaleza en todo su conjunto.

Por lo tanto nos corresponde respetarnos entre todos y todas y hacer lo mismo con la naturaleza porque somos parte de ella y todos los recursos que nos proporciona y la labor que ejecuta son imprescindibles para la vida de todos los seres.

Finalmente, con base en el tema expuesto, se concluye que las mujeres juegan una función importante en el mundo contemporáneo; es primordial la relación de las mujeres con la naturaleza lo que aporta una mirada sobre las necesidades sociales, desde el rescate de la cultura del cuidado como inspiración para pensar una sociedad sostenible.

Esto también se ve reflejado en los pueblos indígenas y en especial en las mujeres pertenecientes a estas comunidades, las cuales hacen una labor importante para preservar la naturaleza, dado que cada pueblo indígena tradicionalmente determina sus formas de vida desde su cosmovisión, y es ahí donde se hace importante retomar el rol de las mujeres para mantener dichas formas de vida en lo que respecta.

En el siglo XXI el ecofeminismo tiene mucho que contribuir y al parecer lo va seguir haciendo, los humanos sean hombres o mujeres, por igual tendremos que enfrentaros a una restructuración del pensamiento, de la cultura, de la visión de mundo, para así lograr 
Hegemonia - Revista Eletrônica de Relações Internacionais do Centro Universitário Unieuro ISSN: 1809-1261

UNIEURO, Brasília, número 20, Janeiro-Junho de 2017, pp. 50-64.

alcanzar un medio ambiente sustentable, donde se pueda vivir y convivir con la naturaleza de iguales y no sobre.

Referencias Bibliográficas

AGARWAL, Bina, "El debate sobre género y medio ambiente: lecciones de la India", en Vázquez García y Velázquez Gutiérrez, 1998.

BEDREGAL, Teresa, Ecofeminismo, Biología y Teoría Feminista, Agenda de las mujere, Argentina, 2005.

BRAIDOTTI, Rosi, "Mujeres, medio ambiente y desarrollo sustentable, Surgimiento del tema y diversas aproximaciones", en Verónica Vázquez García y Margarita Velázquez Gutiérrez (coords.), Miradas al futuro, PUEG/CRIM/CP, México, 1998.

COVA, Santana Nancy, El Ecofeminismo Latinoamericano, Las Mujeres y la Naturaleza como Simbolos, Cifra Nueva, Universidad de los Andes-Trujillo, 2005.

Duque, Elena, "Entrevista a Alicia Puleo: claves del ecofeminismo", Mujeres en la Red, El Periódico feminista, 2009.

ESCOBAR, Elsa, ESCOBAR, Pía, PAZMIÑO, Aracely, ULLOA, Astrid (Editoras), Las mujeres indígenas en los escenarios de la biodiversidad, UICN Fundación Natura Colombia, ICANH, 2005.

ESPINOZA, Frida, "Mujer y Naturaleza: definiciones de Ecofeminismo," In, Planeta Capital, Revista Rufián, vol. No 10, Santiago de Chile, 2012.

FERNANDEZ, Alex, Feminismo ecológico. Eroski Consumer, 2012 Gebara, Ivone, "Intuiciones ecofeministas, ensayo para repensar el conocimiento y la religión”, Editorial Trotta, Madrid, 2000. 
Hegemonia - Revista Eletrônica de Relações Internacionais do Centro Universitário Unieuro ISSN: 1809-1261

UNIEURO, Brasília, número 20, Janeiro-Junho de 2017, pp. 50-64.

LEFF, Enrique, El Retorno del Orden Simbólico, Racionalidad Ambiental, La reapropiación social de la naturaleza, Siglo XI Editores, 2004.

LEÒN, Magdalena: Cambiar la economía para cambiar la vida, Desafíos de una economía para la vida, Alberto Acosta y Esperanza Martínez (comp.): El buen vivir, Una vía para el desarrollo, Abya Yala, Quito, 2009.

Merchant, C. The death of nature. En M. Zimmerman, J. Baird y G, Sessions, Environmental philosophy, Prentice Hall, Nueva Jersey, 1993.

PULEO, Alicia, Libertad, igualdad, sostenibilidad, Por un ecofeminismo ilustrado, Universidad de Valladolid, 2008.

ULLOA, Astrid, Concepciones de la naturaleza en la antropología actual IN Cultura y Naturaleza: Aproximaciones a propósito del bicentenario de la independencia de Colombia. Leonardo Montenegro Martínez (Editor), Bogotá: Jardín Botánico de Bogotá, José Celestino Mutis, 2011.

ULLOA, Astrid, La construcción del nativo ecológico, Complejidades, paradojas y dilemas de la relación entre los movimientos indígenas y el ambientalismo en Colombia, Instituto Colombiano de Antropología e Historia, Colciencias, Bogotá, 2004.

ULLOA, Astrid, Participación politica y ambiental, Mujeres indigenas: dilemas de género y etnicidad en los escenarios latinoamericanos, Universidad Nacional de Colombia, Bogotá, 2007.

VEUTHEY, Sandra, "Ecuador: los conflictos contra la cría de camarones contribuyen a la emancipación de las mujeres", Boletín n 142, WRM, 2009. 